\title{
NSF's ChemMatCARS: A Delicate Third Generation Synchrotron Radiation User facility for Chemical and Materials Crystallography
}

\author{
Yu-Sheng Chen ${ }^{a}$, Suyin Wang ${ }^{a}$, Adam Stasha, \\ ${ }^{a}$ NSF's ChemMatCARS, the University of Chicago, 9700 S. Cass Ave, Lemont, IL 60439
}

Located at the Advanced Photon Source (APS), Argonne National Laboratory (ANL). NSF's ChemMatCARS addresses the need in the U.S. for facilities and expertise in synchrotron X-ray studies of advanced chemical and materials crystallography. More than 140 independent research groups performed X-ray experiments at NSF's ChemMatCARS facilities during 2014-2018 to investigate science relevant to programs in the NSF Divisions of Chemistry and Materials Research. X-ray diffraction experiments at NSF's ChemMatCARS advance our knowledge of the atomic and molecular-level processes that determine the chemistry and materials properties of vitally important crystalline solids, liquids, and interfaces. In advanced crystallography, the program has performed in structural dynamics that promise to achieve an atomic-level understanding of the fundamental photo-physical processes in photoresponsive materials. Resonant diffraction can reveal the redox distribution throughout a catalyst cluster, enabling the design of new catalysts with atomic precision as the result of the atomic-level understanding of how chemical reactions are driven. Temperature and pressure dependent phase transition of the crystalline materials can be easily obtained from the study of the crystalline materials under extreme conditions (low/high temperature, low/high pressure). Combining both can aid in understanding crystalline materials phase diagrams, which can provide a piece of very useful information in material science. A new initiative in 3-dimensional pair distribution function measurements will provide detailed and quantitative information on defects, disorder, and lattice dynamics in single crystals.

The forefront capabilities of the instrumentation and the expertise of NSF's ChemMatCARS scientists have led to a level of scientific productivity and beamline utilization that ranks among the top APS beamlines. A number of different software has been developed for the advanced crystallographic program and the hardware setup will be present in the talk.

Finally, the development of a second beamline at NSF's ChemMatCARS sector enables the development of new techniques and new areas of inquiry. Serial X-ray crystallography of small molecules will be developed to study the structural dynamics of irreversible chemical and physical reactions and processes, which have proven challenging to characterize on the atomic scale. Low energy $X$-rays on the new beamline will extend the capabilities of resonant diffraction to provide complete coverage of $3 \mathrm{~d}$ and $4 \mathrm{~d}$ transition metals. Combined spectroscopy and X-ray diffraction will occur in the proposed new beamline.

\section{Email Contact: yschen@cars.uchicago.edu}

